\title{
BMJ Open Does non-invasive ventilation change metabolic markers in children with obstructive sleep apnoea? A systematic review and meta-analysis study protocol
}

\author{
Christopher Gerdung (D) , , ${ }^{1,2}$ Sara Rodriguez-Lopez (D) , ${ }^{1,2}$ Stefan Palkowski, ${ }^{1,2}$ \\ Diana Keto-Lambert, ${ }^{3,4}$ Meghan Sebastianski, ${ }^{3,4}$ Maria Luisa Castro Codesal (D) 1,2
}

To cite: Gerdung C, RodriguezLopez S, Palkowski S, et al. Does non-invasive ventilation change metabolic markers in children with obstructive sleep apnoea? A systematic review and meta-analysis study protocol. BMJ Open 2020;10:e039655. doi:10.1136/ bmjopen-2020-039655

- Prepublication history and additional material for this paper are available online. To view these files, please visit the journal online (http://dx.doi. org/10.1136/bmjopen-2020039655).

Received 21 April 2020 Revised 26 June 2020 Accepted 06 July 2020

Check for updates

(C) Author(s) (or their employer(s)) 2020. Re-use permitted under CC BY-NC. No commercial re-use. See rights and permissions. Published by BMJ.

${ }^{1}$ Pediatrics, University of Alberta Faculty of Medicine and Dentistry, Edmonton, Alberta, Canada

2Stollery Children's Hospital, Edmonton, Alberta, Canada ${ }^{3}$ Alberta Strategy for PatientOriented Research (SPOR) Knowledge Translation Platform, University of Alberta, Edmonton, Alberta, Canada

${ }^{4}$ University of Alberta Faculty of Medicine and Dentistry Department of Pediatrics, Edmonton, Alberta, Canada

Correspondence to

Dr Maria Luisa Castro Codesal; castroco@ualberta.ca

\section{ABSTRACT}

Introduction Obstructive sleep apnoea (OSA) is not only common within paediatrics but is associated with critical childhood metabolic morbidity such as obesity, cardiovascular disease and glucose tolerance impairment. Increasing evidence suggests an association between childhood OSA and metabolic syndrome such as markers of cardiovascular disease, systemic hypertension, glucose intoleranceand increased lipid profile. Recent studies have targeted changes in metabolic markers in children using non-invasive ventilation (NIV) but no systematic reviews are available to summarise this emerging evidence. The purpose of this systematic review is to provide systematic synthesis of the evidence on the effect of NIV use on metabolic markers in children with OSA.

Methods and analysis A systematic search of electronic databases and grey literature will include paediatric interventional studies (random controlled trials, cohort studies) with and without a comparison group. Two reviewers will independently undertake the two step process of title/abstract and full-text screening. Data will be extracted and assessed, with aggregate data being reported. When the data allow, meta-analysis will be performed.

Ethics and dissemination There are no ethical concerns with this systematic review, as data have previously been published. This review will inform clinicians taking care of children with OSA and obesity/metabolic syndrome about the potential effects of NIV therapies on metabolic markers and has the potential to change the approach to childhood OSA and obesity. Results of this systematic review will be submitted for dissemination in abstract and manuscript form.

\section{INTRODUCTION}

Obstructive sleep apnoea (OSA) is common in children with an estimated prevalence of $5.7 \% .^{1}$ OSA contributes to childhood morbidity, including impaired cognition, neurodevelopmental delay, cardiometabolic dysfunction and reduced quality of life. ${ }^{2}$ The metabolic consequences of OSA are well known among adult populations, with a clearly demonstrated association between
Strengths and limitations of this study

- We will use comprehensive systematic review and meta-analysis methods to summarise the evidence on the efficacy of a respiratory intervention for an increasing health problem in children worldwide: obesity and metabolic syndrome.

- Methodological experts and paediatric clinical specialists have been consulted to ensure that our search strategy captures all potential metabolic markers that might be impacted by non-invasive ventilation.

- We will attempt a meta-analysis when there is enough homogeneity among populations and measured outcomes.

- Limited appraisal of the grey literature will be performed.

OSA and metabolic syndrome, including systemic hypertension, cardiovascular risk, dyslipidaemia, glucose control impairment and systemic inflammation. ${ }^{34}$ The literature examining the association between metabolic syndrome in children has shown a similar relationship with an emerging body of the literature demonstrating a greater risk of hypertension, dyslipidaemia and glucose control in obese children and youths with OSA. $^{5-8}$

The first line therapy for the treatment of OSA in children is adenotonsillectomy. ${ }^{29}$ In both obese and non-obese children, surgical intervention can improve cardiorespiratory parameters in sleep studies including Apnoea Hypopnoea Index (AHI) and oxygen saturation values. Surgical management has also been shown to have positive effects on systemic inflammation and dyslipidaemia. ${ }^{10-12}$ However, residual symptoms of OSA after adenotonsillectomy have been shown to be present in up to half of children, which is particularly true in children with obesity. ${ }^{13-15}$ 
Given the persistence of OSA after surgery, the metabolic consequences are likely to persist. Non-invasive ventilation (NIV), including continuous positive airway pressure (CPAP) and bi-level positive airway pressure (BPAP), is the recommended therapy for children with OSA who are not surgical candidates, or for children with persistent OSA post-surgery. Initiation of NIV has been shown to have significant effects on nocturnal symptoms of OSA and daytime outcomes such as daytime sleepiness, attention, academic performance and quality of life. ${ }^{16-20}$ While previous systematic reviews and meta-analysis have summarised the impact of NIV in metabolic markers in the adult population, including hypertension cardiovascular outcomes, glucose control, lipid profile, systemic inflammation and even mortality, ${ }^{19} 21-29$ similar systematic reviews are not available in paediatric populations. The purpose of this systematic review is to provide a rigorous overview of the evidence describing the effect of NIV use on metabolic markers in children with OSA.

\section{METHODS AND ANALYSIS \\ Study design}

The methodology of this systematic review and metaanalysis follows the recommendations made in the Preferred Reporting Items for Systematic Reviews and Meta-Analysis Protocols 2015 statement. ${ }^{30}$ Approval from the National Institute for Health Research PROSPERO registry is pending.

\section{Search strategy}

An information specialist, systematic review methodologist, and clinical experts in paediatric respiratory medicine, pediatric sleep medicine, and paediatric metabolic syndrome have established a collaborative team to develop a comprehensive search strategy and determined the appropriate information sources. The search strategy includes terms for NIV used in a previously published scoping review, ${ }^{31}{ }^{32}$ a published filter for paediatric populations $^{31} 32$ and addresses the following key concepts: effects on oxidative stress and markers of systemic inflammation, cardiovascular outcomes, changes in weight/body mass index, lipid profile, liver enzymes, glucose control and markers of insulin resistance, renal function and other metabolic markers (see online supplementary appendix A). ${ }^{31}$ Information sources will include Ovid Medline, Ovid Embase, CINAHL via EbscoHOST, Wiley Cochrane Library (including the Cochrane Database of Systematic Reviews, the Cochrane Central Register of Controlled Trials, the Database of Abstracts of Reviews of Effects, the Health Technology Assessment Database and the NHS Economic Evaluation Database) and PROQUEST Theses and Dissertations. No language restrictions will be applied to the search strategy. Letters, editorials, commentaries and non-intervention studies will be excluded. Reference lists of all studies included in this systematic review will be scanned to identify further relevant studies not detected by the search strategy.
Published abstracts from 2018 to 2019 from the following conferences will be screened: American Thoracic Society Conference, Canadian Thoracic Society Conference, CHEST Congress, European Respiratory Society, Sleep Meeting (see online supplementary appendix B). The search of the above information sources is planned to occur on 30 April 2020.

\section{Eligibility criteria}

Types of participants/population

Studies including children (newborn to age 18 years) will be included. Studies across ages with disaggregated paediatric data will also be included. Eligible studies must pertain to children with OSA or other sleep-related breathing disorders as defined in the International Classification of Sleep Disorders, including obstructive sleep apnoea and sleep-related hypoventilation.

\section{Types of interventions}

Included studies must contain information on NIV (CPAP or BPAP) use. We will include studies irrespective of the length of NIV usage.

\section{Type of outcomes}

Eligible studies must include data related to metabolic markers, with no outcome restrictions. This includes but is not limited to: effects on oxidative stress and markers of systemic inflammation, cardiovascular outcomes, changes in weight/body mass index, lipid profile, liver enzymes, glucose control and markers of insulin resistance, renal function and other metabolic markers. Inflammatory markers, cardiovascular outcomes and other outcomes of metabolic syndrome are described in online supplementary appendix $\mathrm{A}$.

\section{Types of studies}

Interventional studies will be considered for inclusion including randomised and non-randomised clinical trials (RCTs and non-RCTs), controlled before-after studies. No other study designs will be considered for inclusion.

\section{Exclusion}

Qualitative and mixed methods research will be excluded, as will case series, case reports and non-specified grey literature.

\section{Study records}

Data management

Records from searches will be imported into an EndNote library (EndNote V.X9, Clarivate Analytics) and duplicates will be removed. The library will be duplicated for independent screening.

\section{Selection process}

Two independent reviewers (MCC, CAG) will screen titles and abstracts of retrieved articles for eligibility based on the inclusion criteria. Full text will be retrieved for all potentially relevant articles, which will be reviewed independently by the two reviewers, and studies that meet the 
eligibility criteria will be included in the review. Discrepancies between the reviewers will be resolved through discussion. Reasons for exclusion will be recorded at the full-text review level.

\section{Data collection process}

Data extraction will be completed by one reviewer using a pre-designed standardised form and entered into RedCap Database. ${ }^{33} 34$ Data extraction will be verified by a second reviewer for a sample of $20 \%$ of the studies with acceptance if agreement in at least $80 \%$ of the data. Duplicate data from the same data set published in different manuscripts will be removed. Data extraction is expected to be complete by 1 October 2020 .

\section{Data synthesis}

Once the information has been extracted, we will present findings including the numerical analysis of the number, publication type, publication year and country of publication of the studies included in the review. We will also present a narrative description of the study design, participant characteristics, sample size, NIV type, control group description, outcomes measures and follow-up duration. We will use this information to establish subcategories of studies which may include groupings based on age (ie, infants, children and adolescents), intervention type (ie, CPAP, bi-level) and outcomes (eg, oxidative stress, obesity, dyslipidaemia, liver disease, glucose intolerance, cardiovascular, renal and other metabolic diseases).

\section{Quality assessment and risk of bias}

Risk of bias will be assessed by two independent reviewers (MCC, CAG) specific to the study methodology. Tools will include the Quality Assessment Tool ${ }^{35}$ and Cochrane risk of bias tool. ${ }^{36}$ Discrepancies in decisions will be resolved through discussion.

The reviewers will also use the Grading of Recommendations, Assessment, Development and Evaluations to assess the evidence at the outcome level. ${ }^{37}$ This will include assessing for risk of bias, imprecision, inconsistency, indirectness and publication bias.

\section{Meta-analysis}

Meta-analysis will be attempted for outcomes with enough homogeneity among the study populations. Homogeneity criteria will include an AHI $<5$ events/hour to define mild obstructive sleep apnoea, 5-10 events/hour for moderate and $>10$ for severe sleep disorder breathing as per the American Academy of Sleep Medicine. ${ }^{38}{ }^{39}$ In addition, nocturnal hypoxemia will be defined by sleep time with oxygen saturations below $90 \%$ over $5 \%$ and nocturnal hypoventilation by over $25 \%$ sleep time carbon dioxide levels above $50 \mathrm{~mm} \mathrm{Hg} .{ }^{40}$ The information will be subcategorised to include grouping based on outcome (changes in the same metabolic marker). For non-randomised studies, we will combine effect estimates from studies adjusted by confounding rather than raw data if available. RCTs will be analysed separately if available. Risk of bias will be considered and explanations for heterogeneity will be pursued including publication bias. Graphics of pooled data (ie, forest plots) will be provided. Most outcome data are expected to be continuous. Heterogeneity in the pooled data will be minimised, by pooling data based on outcome, as well as the indication for NIV. In the event that pooled data remain heterogeneous within these pooled groups, a narrative description will be included. If the data allow, we will analyse the data with respect to NIV adherence and the length of time wearing NIV.

\section{Patient and public involvement}

Patients and public were not involved in the design of this study. Dissemination of the information may include targeting patients and their care providers, as the results of the study may inform us of the effect of NIV on metabolic outcomes in children with sleep-related breathing disorders.

\section{Ethics and dissemination}

There are no ethical concerns with this systematic review, as data have previously been published. This review will inform clinicians taking care of children with OSA and obesity/metabolic syndrome about the potential effects of NIV therapies on metabolic markers and help plan for necessary assessment of this group of children in the clinical practice. This information has the potential to change the approach to childhood OSA and obesity and therefore impact future clinical guidelines at national and international level. Results of this systematic review will be submitted for dissemination in abstract and manuscript form at multiple levels to target general paediatricians and paediatric specialists involved in the care of these patients including paediatric respiratory and sleep specialists, endocrinologists, cardiologists and nephrologists. The results of this systematic review will be of interest for general audience, which can be reached through infographics, videos and TED talks to be distributed through social media.

Contributors MLCC, CG and SR-L were involved in idea generation of this study. MLCC, CG, SR-L, SP, DK-L and MS were involved in study design and protocol development. MS and DK-L developed the search strategies and performed literature reviews. MLCC and CG were involved with review of literature for inclusion into the study.

Funding This work was supported by the Alberta Strategy for Patient-Oriented Research (SPOR) SUPPORT Unit Knowledge Translation Platform, which is funded by Alberta Innovates and the Canadian Institutes of Health Research.

Competing interests None declared.

Patient consent for publication Not required.

Provenance and peer review Not commissioned; externally peer reviewed.

Open access This is an open access article distributed in accordance with the Creative Commons Attribution Non Commercial (CC BY-NC 4.0) license, which permits others to distribute, remix, adapt, build upon this work non-commercially, and license their derivative works on different terms, provided the original work is properly cited, appropriate credit is given, any changes made indicated, and the use is non-commercial. See: http://creativecommons.org/licenses/by-nc/4.0/.

\section{ORCID iDs}

Christopher Gerdung http://orcid.org/0000-0003-0096-3993

Sara Rodriguez-Lopez http://orcid.org/0000-0003-0543-4855 
Maria Luisa Castro Codesal http://orcid.org/0000-0002-1079-2502

\section{REFERENCES}

1 Marcus CL, Brooks LJ, Draper KA, et al. Diagnosis and management of childhood obstructive sleep apnea syndrome. Pediatrics 2012;130:e714-55.

2 Marcus $\mathrm{CL}$, Moore RH, Rosen CL, et al. A randomized trial of adenotonsillectomy for childhood sleep apnea. $N$ Engl J Med 2013;368:2366-76.

3 Kong D-L, Qin Z, Wang W, et al. Association between obstructive sleep apnea and metabolic syndrome: a meta-analysis. CIM 2016:39:161-72.

4 Xu S, Wan Y, Xu M, et al. The association between obstructive sleep apnea and metabolic syndrome: a systematic review and metaanalysis. BMC Pulm Med 2015;15:105

5 Patinkin ZW, Feinn R, Santos M. Metabolic consequences of obstructive sleep apnea in adolescents with obesity: a systematic literature review and meta-analysis. Child Obes 2017;13:102-10.

6 Koren D, Gozal D, Philby MF, et al. Impact of obstructive sleep apnoea on insulin resistance in nonobese and obese children. Eur Respir J 2016;47:1152-61.

7 Redline S, Storfer-Isser A, Rosen CL, et al. Association between metabolic syndrome and sleep-disordered breathing in adolescents. Am J Respir Crit Care Med 2007;176:401-8.

8 Verhulst SL, Schrauwen N, Haentjens D, et al. Sleep-disordered breathing and the metabolic syndrome in overweight and obese children and adolescents. J Pediatr 2007;150:608-12.

9 Alsubie HS, BaHammam AS. Obstructive sleep apnoea: children are not little adults. Paediatr Respir Rev 2017;21:72-9.

10 Kheirandish-Gozal L, Gileles-Hillel A, Alonso-Álvarez ML, et al. Effects of adenotonsillectomy on plasma inflammatory biomarkers in obese children with obstructive sleep apnea: a community-based study. Int J Obes 2015;39:1094-100.

11 Gozal D, Capdevila OS, Kheirandish-Gozal L. Metabolic alterations and systemic inflammation in obstructive sleep apnea among nonobese and obese prepubertal children. Am J Respir Crit Care Med 2008;177:1142-9.

12 Waters KA, Sitha S, O'brien LM, et al. Follow-Up on metabolic markers in children treated for obstructive sleep apnea. Am J Respir Crit Care Med 2006;174:455-60.

13 Scheffler P, Wolter NE, Narang I, et al. Surgery for obstructive sleep apnea in obese children: literature review and meta-analysis. Otolaryngol Head Neck Surg 2019;160:985-92.

14 Kang K-T, Koltai PJ, Lee C-H, et al. Lingual tonsillectomy for treatment of pediatric obstructive sleep apnea: a meta-analysis. JAMA Otolaryngol Head Neck Surg 2017;143:561-8.

15 Rivero A, Durr M. Lingual tonsillectomy for pediatric persistent obstructive sleep apnea: a systematic review and meta-analysis. Otolaryngol Head Neck Surg 2017;157:940-7.

16 Beebe DW, Byars KC. Adolescents with obstructive sleep apnea adhere poorly to positive airway pressure (PAP), but Pap users show improved attention and school performance. PLoS One 2011;6:e16924.

17 Marcus CL, Rosen G, Ward SLD, et al. Adherence to and effectiveness of positive airway pressure therapy in children with obstructive sleep apnea. Pediatrics 2006;117:e442-51.

18 Marcus CL, Radcliffe J, Konstantinopoulou S, et al. Effects of positive airway pressure therapy on neurobehavioral outcomes in children with obstructive sleep apnea. Am J Respir Crit Care Med 2012;185:998-1003.

19 Patil SP, Ayappa IA, Caples SM, et al. Treatment of adult obstructive sleep apnea with positive airway pressure: an American academy of sleep medicine systematic review, meta-analysis, and grade assessment. J Clin Sleep Med 2019;15:301-34.
20 Uong EC, Epperson M, Bathon SA, et al. Adherence to nasal positive airway pressure therapy among school-aged children and adolescents with obstructive sleep apnea syndrome. Pediatrics 2007;120:e1203-11.

21 Martinez-Garcia MA, Campos-Rodriguez F, Javaheri S, et al. Pro: continuous positive airway pressure and cardiovascular prevention. Eur Respir J 2018;51:1702400.

22 Masa JF, Corral-Peñafiel J. Should use of 4 hours continuous positive airway pressure per night be considered acceptable compliance? Eur Respir J 2014;44:1119-20.

23 Weaver TE, Maislin G, Dinges DF, et al. Relationship between hours of CPAP use and achieving normal levels of sleepiness and daily functioning. Sleep 2007;30:711-9.

24 Wolf J, Narkiewicz K. Optimizing the management of uncontrolled/ resistant hypertension. The importance of sleep apnoea syndrome. Curr Vasc Pharmacol 2017;16:44-53.

25 Lei Q, Lv Y, Li K, et al. Effects of continuous positive airway pressure on blood pressure in patients with resistant hypertension and obstructive sleep apnea: a systematic review and meta-analysis of six randomized controlled trials. J Bras Pneumol 2017;43:373-9.

26 Zhu B, Ma C, Chaiard J, et al. Effect of continuous positive airway pressure on glucose metabolism in adults with type 2 diabetes: a systematic review and meta-analysis of randomized controlled trials. Sleep Breath 2018;22:287-95.

27 McEvoy RD, Kohler M. Con: continuous positive airway pressure and cardiovascular prevention. Eur Respir J 2018:51:1702721.

28 Lee CP, Kushida CA, Abisheganaden JA. Epidemiological and pathophysiological evidence supporting links between obstructive sleep apnoea and type 2 diabetes mellitus. Singapore Med $J$ 2019;60:54-6.

29 Labarca G, Reyes T, Jorquera J, et al. CPAP in patients with obstructive sleep apnea and type 2 diabetes mellitus: systematic review and meta-analysis. Clin Respir J 2018;12:2361-8.

30 Moher D, Shamseer L, Clarke $M$, et al. Preferred reporting items for systematic review and meta-analysis protocols (PRISMA-P) 2015 statement. Syst Rev 2015;4:1.

31 Castro Codesal ML, Featherstone R, Martinez Carrasco C, et al. Long-term non-invasive ventilation therapies in children: a scoping review protocol. BMJ Open 2015;5:e008697.

32 Castro-Codesal ML, Dehaan K, Bedi PK, et al. Longitudinal changes in clinical characteristics and outcomes for children using long-term non-invasive ventilation. PLoS One 2018;13:e0192111.

33 Harris PA, Taylor R, Minor BL, et al. The REDCap consortium: building an international community of software platform partners. $J$ Biomed Inform 2019;95:103208.

34 Harris PA, Taylor R, Thielke R, et al. Research electronic data capture (REDCap)-a metadata-driven methodology and workflow process for providing translational research informatics support. J Biomed Inform 2009;42:377-81.

35 Thomas H. Quality assessment tool for uantitative studies. effective public health Practicde project, 2003. Available: https://www.ephpp. $\mathrm{ca}$ /quality-assessment-tool-for-quantitative-studies/

36 Higgins JPT, Altman DG, Gøtzsche PC, et al. The Cochrane collaboration's tool for assessing risk of bias in randomised trials. BMJ 2011;343:d5928.

37 Guyatt G, Oxman AD, Akl EA, et al. GRADE guidelines: 1. Introduction-GRADE evidence profiles and summary of findings tables. J Clin Epidemiol 2011;64:383-94.

38 Dehlink E, Tan H-L. Update on paediatric obstructive sleep apnoea. J Thorac Dis 2016;8:224-35.

39 DeHaan KL, Seton C, Fitzgerald DA, et al. Polysomnography for the diagnosis of sleep disordered breathing in children under 2 years of age. Pediatr Pulmonol 2015;50:1346-53.

40 Berry RB, Albertario CL, HS M. The AASM manual for the scoring of sleep and assoicated events: rules, terminology and technical specifications. version 2.5. Darien, IL: American Academy of Sleep Medicine, 2018. 